\title{
Solution Properties of Synthetic Polypeptides. XIII. Dimensions of Interrupted Helices of $\operatorname{Poly}(\gamma$-benzyl L-glutamate)
}

\author{
Takashi Norisuye, Akio Teramoto, and Hiroshi Fujita \\ Department of Polymer Science, Osaka University, Toyonaka 560, Japan.
}

(Received June 27, 1972)

\begin{abstract}
The helix-coil transition of poly( $\gamma$-benzyl L-glutamate) in dichloroacetic acid containing 8.3-wt \% cyclohexanol was studied by light-scattering and optical rotation measurements. Data for the mean-square radii of gyration $\left\langle S^{2}\right\rangle$ were analyzed by the method of Teramoto, et al., with the aid of the transition parameters estimated from the optical rotation data, yielding $1.53 \pm 0.1 \AA$ for the pitch per monomeric residue in helical sections. This value is in excellent agreement with that of $1.5 \AA$ expected for the $\alpha$-helix, and confirms the recent result of Okita, et al., for poly-[ $N^{5}$-(3-hydroxypropyl) L-glutamine]. Light-scattering measurements were also made with solutions of a helicogenic solvent, dimethylformamide (DMF). The values of the pitch calculated directly from $\left\langle S^{2}\right\rangle$ data in DMF were smaller than $1.5 \AA$ and showed a trend to decrease slightly with increasing molecular weight, being in accordance with data of previous workers. This trend was successfully interpreted in terms of the interrupted helix model when the experimental values of the transition parameters for the helicogenic solvents was used.
\end{abstract}

KEY WORDS Helix-Coil Transition / Light Scattering / Interrupted Helix / Poly( $\gamma$-benzyl L-glutamate) / Mean-Square Radius of Gyration / Flexibility of Polypeptides in Helicogenic Solvents /

In Part IX of this series, with light-scattering and optical rotatory dispersion (ORD) data for poly-[ $N^{5}$-(3-hydroxypropyl)L-glutamine] (PHPG) in mixtures of water and methanol, Okita, et al., ${ }^{1}$ demonstrated for the first time that molecular dimensions of interrupted helices can be described quantitatively by the theory due originally to Nagai, ${ }^{2}$ although, in actually, its approximate form deduced by Teramoto, et al., ${ }^{3}$ was used. We here report a similar work performed with poly $(\gamma$-benzyl L-glutamate) (PBLG) in order to attest the validity of Nagai's theory. For this purpose, we selected dichloroacetic acid (DCA) containing 8.3-wt\% cyclohexanol (CHL) as the solvent. The reason is based on the finding by Strazielle, et al., ${ }^{4}$ and is twofold: (1) DCA and CHL have almost identical refractive indices so that light-scattering measurements in their mixtures escape from the effect of preferential adsorption, and (2), at the solvent composition chosen, the conversion of PBLG from coil to helix may occur at temperatures convenient for light-scattering and polarimetric measurements.

Dimethylformamide (DMF) and ethylene dichloride (EDC) are familiar helicogenic solvents for PBLG. If the $\alpha$-helix formed by the PBLG chain in such a solvent is intact and rigid, light-scattering determination of the mean-square radius of gyration, $\left\langle S^{2}\right\rangle$, should yield a value of $1.5 \AA$ for the pitch per monomeric residue, $h$, regardless of the molecular weight of the sample used. Measurements by previous investigators, ${ }^{5-9}$ however, did not always substantiate this prediction, showing that the values of $h$ computed from the measured $\left\langle S^{2}\right\rangle^{1 / 2}$ divided by the degree of polymerization were smaller than $1.5 \AA$ and decreased monotonically with increasing molecular weight. This phenomenon has been attributed either to a certain flexibility or an imperfection of the helix..$^{10} \mathrm{We}$ felt it worthwhile to provide further experimental data on the molecular weight dependence of $h$, and conducted measurements of $\left\langle S^{2}\right\rangle$ for 
several well-fractionated samples of PBLG in DMF. The results obtained are also reported in this paper.

\section{EXPERIMENTAL}

\section{Polymer Samples and Solutions}

Seven samples of PBLG prepared and carefully fractionated in this laboratory were chosen for the present work. Three of them, designated below as E-1, E-3, and A-6, had been used for the study reported in Part $X^{11}$ Each sample was freeze-dried from a dioxane solution and further dried overnight in a vacuum oven at $40^{\circ} \mathrm{C}$.

Mixtures of DCA and CHL were quite unstable, regardless of the presence or absence of the polypeptide solute. This was presumably due to either oxidation of CHL or ester formation between the two components. Hence, we made up each test solution in such a way that first the polymer was dissolved in DCA and then CHL was added to the desired solvent composition, and then attempted physical measurements on it as quickly as possible.

\section{Solvents}

CHL was fractionally distilled under reduced nitrogen atmosphere, bp $68.0^{\circ} \mathrm{C}(16 \mathrm{~mm})$, and DCA and DMF were purified as described previously. ${ }^{11}$

The refractive indices of the purified solvents were measured, yielding 1.439 at $436 \mathrm{~nm}$ and 1.431 at $546 \mathrm{~nm}$ for $\mathrm{DMF}$ at $25^{\circ} \mathrm{C}$, and 1.467 , 1.466 , and 1.464 at $546 \mathrm{~nm}$ for the DCA-CHL mixture (DCA containing 8.3-wt\% of CHL) at $20^{\circ}, 25^{\circ}$, and $30^{\circ} \mathrm{C}$, respectively. The densities of this mixture at $20^{\circ}, 30^{\circ}$, and $40^{\circ} \mathrm{C}$ were $1.484,1.471$, and $1.458 \mathrm{~g} / \mathrm{ml}$, respectively. Values of the refractive index and density of the mixture at other temperatures were estimated by either extrapolation or interpolation of these experimental values.

\section{Optical Rotation Measurement}

Specific rotation for light of wavelength 546 $\mathrm{nm}$, denoted below by $[\alpha]_{546}$, was measured for samples E-1, E-3, E-4, and A-6 in the DCACHL mixture at various temperatures in the range $10-30^{\circ} \mathrm{C}$. The measured values of $[\alpha]_{546}$ were then converted to the helical contents, $f_{N}$, by using the empirical relation ${ }^{12}$

$$
f_{N}=\left([\alpha]_{546}+21.5\right) / 41.2
$$

where the subscript $N$ to $f$ denotes the degree of polymerization of the sample.

Specific Refractive Index Increment $d n / d c$

For a suitably chosen sample this quantity in DMF and in the DCA-CHL mixture was determined at $25^{\circ} \mathrm{C}$ by means of a differential refractometer of the modified Schulz-Cantow type. The values obtained were $0.121_{0}(\mathrm{~m} / \mathrm{g})$ at $436 \mathrm{~nm}$ and $0.117_{1}$ at $546 \mathrm{~nm}$ for DMF, and $0.092_{5}$ at $546 \mathrm{~nm}$ for the DCA-CHL mixture. Further measurements with samples of different molecular weights revealed no detectable dependence of $\mathrm{d} n / \mathrm{d} c$ on molecular weight, both in DMF and in the DCA-CHL mixture. For the reason previously mentioned by Okita, et $a l .{ }^{1}$ therefore, we ignored the copolymer nature of the interrupted helix in the ensuing analysis of light-scattering data.

\section{Light-scattering Photometry}

Except for minor points, the same apparatus and experimental procedures as in our previous studies of this series were employed. For the calibration of the photometer we here assumed the Rayleigh ratio for pure benzene at $25^{\circ} \mathrm{C}$ to be $46.5 \times 10^{-6}$ at $436 \mathrm{~nm}$ and $16.1 \times 10^{-6}$ at 546 $\mathrm{nm},{ }^{13}$ differing from Part $\mathrm{X}$ in which this ratio at $436 \mathrm{~nm}$ had been taken to be $48.5 \times 10^{-6}$. Because of this alternation, the values of weight average molecular weight $\bar{M}_{w}$ reported for samples E-1 and E-3 in Part X were lowered by about $5 \%$ when quoted in the present paper.

For the study of molecular dimensions in DMF we chose samples E-1, F-2, E-2, A-X, and E-3, and carried out measurements at $25^{\circ} \mathrm{C}$. On the other hand, the behavior in the DCA-CHL mixture was examined with samples E-1 and $\mathrm{F}-2$, the temperatures of measurement being varied in the range $10-30^{\circ} \mathrm{C}$.

\section{RESULTS}

\section{Evaluation of Transition Parameters}

Figure 1 shows transition curves $\left([\alpha]_{546} v s\right.$. temperature) for PBLG of different molecular weights in the DCA-CHL mixture. Plots of $f_{N}$ vs. $\bar{N}_{n}^{-1}$ calculated from these curves are 


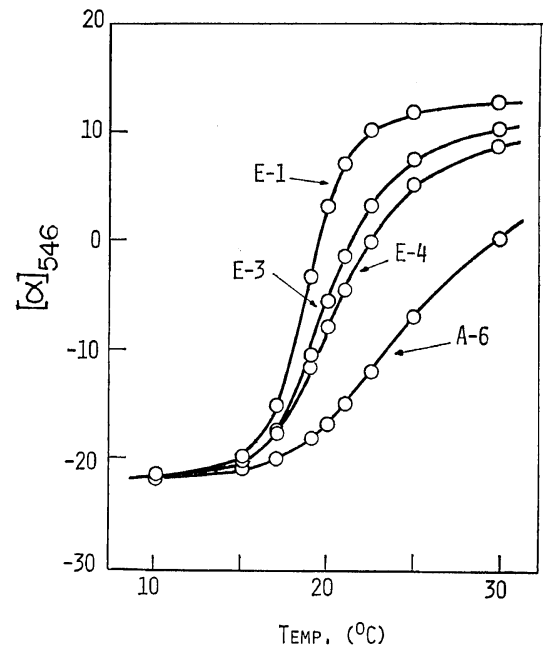

Figure 1. Temperature dependence of specific rotation at $546 \mathrm{~nm},[\alpha]_{546}$, for PBLG in DCA containing 8.3-wt $\%$ CHL as a function of molecular weight.

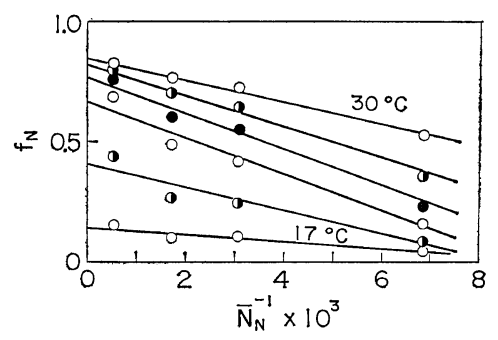

Figure 2. Plots of $f_{N} v s . \bar{N}_{\mathrm{n}}^{-1}$ for PBLG in the DCA-CHL (8-3-wt $\%$ CHL) mixture at 30, 25, $22.5,21,19$, and $17^{\circ} \mathrm{C}$ from top to bottom.

Table I. Numerical values of the transition parameters $f, \sqrt{\sigma}$, and $\ln u$ for PBLG in DCA containing $8.3-w t \%$ cyclohexanol

\begin{tabular}{clcc}
\hline Temp, ${ }^{\circ} \mathrm{C}$ & $f$ & $\sqrt{\sigma} \times 10^{2}$ & $\ln u \times 10^{2}$ \\
\hline 10 & 0 & - & - \\
15 & $0.04_{5}$ & 0.8 & $-3.6^{\circ}$ \\
17 & $0.14_{0}$ & $0.7_{3}$ & $-1.7_{3}$ \\
19 & $0.4_{1}$ & $0.8_{5}$ & $-0.3_{2}$ \\
20 & $0.5_{5}$ & $0.8_{8}$ & $0.1_{8}$ \\
21 & $0.6_{6}$ & $0.8_{7}$ & $0.5_{7}$ \\
22.5 & $0.76_{1}$ & $0.8_{7}$ & $1.0_{6}$ \\
25 & $0.2_{2}$ & $0.8_{9}$ & $1.6_{5}$ \\
30 & $0.84_{5}$ & $1.4_{0}$ & $2.6_{7}$ \\
\hline
\end{tabular}

displayed in Figure 2. Here $\bar{N}_{n}$ is the numberaverage degree of polymerization of the sample.

As expounded in detail in Part $\mathrm{VI},{ }^{14}$ the straight lines fitting the data points in Figure 2 permit the computation of the parameters $f$, $\sqrt{\sigma}$, and $\ln u$ as a function of temperature. Here $u$ is the equilibrium constant for the formation of helix from random coil, $\sigma$ is the cooperativity parameter or the helix-initiation parameter, and $f$ is the value of $f_{N}$ for infinite $N$. Table I summarizes the results of this computation. It is seen that the values of $\sqrt{\sigma}$ obtained are almost constant in the temperature range studied. Similar results for $\sqrt{\sigma}$ were obtained in our previous investigations dealing with PBLG and poly( $\beta$-benzyl L-aspartate) in mixtures of DCA and EDC. ${ }^{11,15} \mathrm{~A}$ value of $860 \pm 80 \mathrm{cal} / \mathrm{mol}$ is obtained for the transition enthalpy at the transition temperature $T_{c}=$ $19.5^{\circ} \mathrm{C}$.

\section{Results from Light-scattering Measurements}

Experience shows that extrapolation of $K c / R_{\theta}$ at fixed scattering angle $\theta$ to zero concentration is facilitated by plotting the square root of $K c / R_{\theta}$ against solute concentration $c$. Here $K$ is the familiar optical constant, and $R_{\theta}$ is the reduced intensity of scattered light. Figure 3 shows plots of this type for sample E-1 in DMF at $25^{\circ} \mathrm{C}$. It is seen that the data points at each $\theta$ can be extrapolated linearly to infinite dilution. In Figure 4, the inverse square root of particle scattering function $P(\theta)$ for five samples in

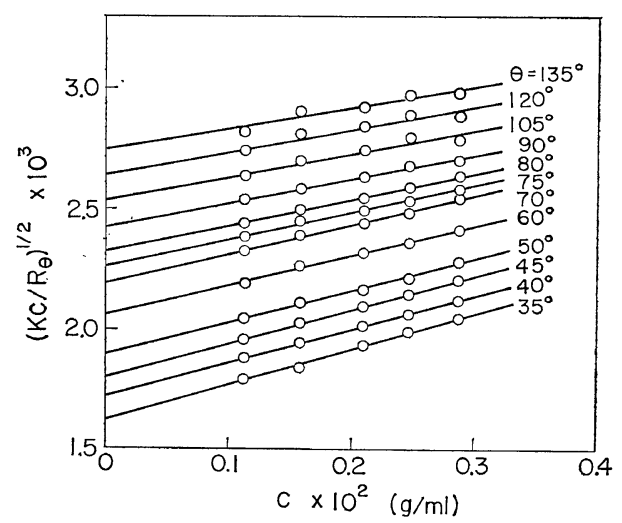

Figure 3. Representative plots of $\left(K c / R_{\theta}\right)^{1 / 2} v s . c$ for sample E-1 in DMF at $436 \mathrm{~nm}$ as a function of scattering angle. 


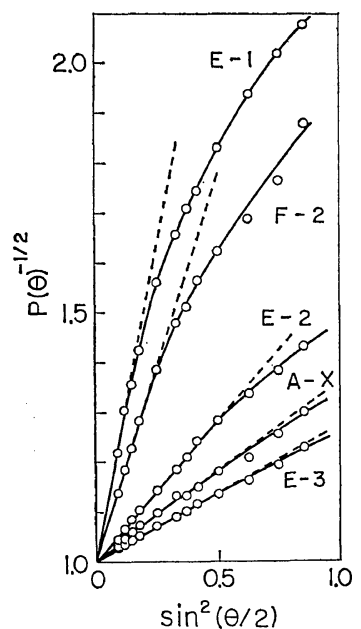

Figure 4. Plots of $P(\theta)^{-1 / 2} v s$. $\sin ^{2}(\theta / 2)$ for PBLG samples in DMF at $436 \mathrm{~nm}$. The dashed line indicates the initial tangent for each curve.

DMF at $25^{\circ} \mathrm{C}$ is plotted against $\sin ^{2}(\theta / 2)$. The initial tangents of the plots for lower molecular weight samples can be determined accurately, since the plots are linear over a fairly wide range of the abscissa. On the other hand, in the plots of higher molecular weight samples E-1 and F-2, only a few points at low scattering angles follow a straight line, making it difficult to determine the initial tangents with certainty.

Under such situation, one will be led to a somewhat underestimated value for the meansquare radius of gyration, $\left\langle S^{2}\right\rangle$. On the contrary, if the data were analyzed in terms of the usual Zimm plot, in which $P(\theta)^{-1}$ is plotted against $\sin ^{2}(\theta / 2)$, one would obtain an $\left\langle S^{2}\right\rangle$ value overestimated to some degree. Based on these considerations, we treated the raw data for samples E-1 and F-2 by the above-mentioned two types of plot, and took the average of the resulting $\left\langle S^{2}\right\rangle$ as the desired one for each.

Data for $K c / R_{0}$ as a function of $c$ can also be analyzed conveniently by plotting their square root against $c$, the weight-average molecular weight, $\bar{M}_{w}$, of the sample and the second virial coefficient of the solution, $A_{2}$, being obtainable from the intercept and initial slope of the resulting plot. Plots of this type for the five samples in DMF at $25^{\circ} \mathrm{C}$ are displayed in Figure 5.

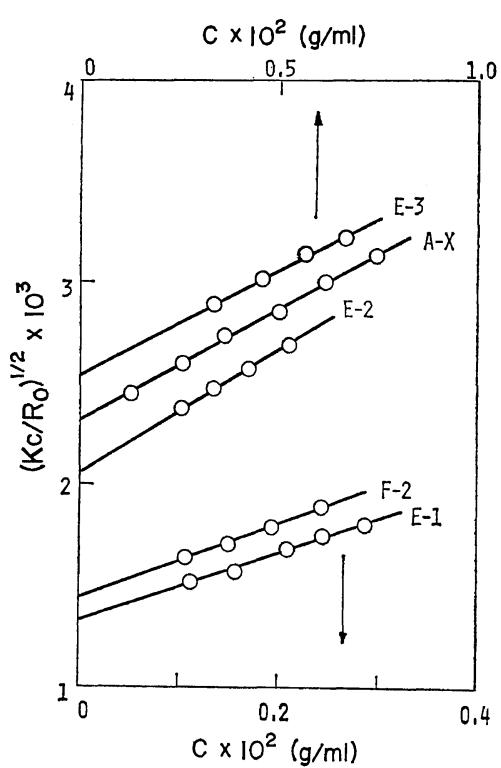

Figure 5. Plots of $\left(K c / R_{0}\right)^{1 / 2} v s . c$ for the indicated samples in DMF at $436 \mathrm{~nm}$. $R_{0}$ denotes the value of $R_{\theta}$ at $\theta=0$.

Table II. Light-scattering data for PBLG in DMF at $25^{\circ} \mathrm{C}$

\begin{tabular}{cccccl}
\hline $\begin{array}{c}\text { Sample } \\
\text { code }\end{array}$ & $\begin{array}{c}\bar{M}_{w} \\
\times \\
10^{-4}\end{array}$ & $\begin{array}{c}\bar{M}_{n} \\
\times \\
10^{-4}\end{array}$ & $\begin{array}{c}A_{2} \times 10^{4}, \mathrm{~m}^{2} \\
\mathrm{mlmol} / \mathrm{g}^{2}\end{array}$ & $\begin{array}{c}\left\langle S^{2}\right\rangle^{1 / 2}, \\
\AA\end{array}$ & $h, \AA$ \\
\hline E-1 & 56.7 & $42.2^{\mathrm{b}}$ & $2.2_{4}$ & $1020 \pm 70$ & $1.36 \pm 0.09$ \\
$\mathrm{~F}-2$ & 47.7 & - & $2.5_{4}$ & $765_{ \pm 15}$ & $1.22 \pm 0.02$ \\
$\mathrm{E}-2$ & 23.7 & - & $2.4_{6}$ & $45_{0}$ & $1.4_{4}$ \\
$\mathrm{~A}-\mathrm{X}$ & 18.8 & - & $2.5_{3}$ & $36_{8}$ & $1.4_{8}$ \\
$\mathrm{E}-3$ & 15.8 & $12.8^{\mathrm{b}}$ & $2.7_{8}$ & $31_{5}$ & $1.5_{1}$ \\
$\mathrm{E}-4$ & $8.0_{8^{\mathrm{a}}}$ & $7.1_{4}^{\mathrm{b}}$ & $3.2^{\mathrm{c}}$ & - & - \\
$\mathrm{A}-6$ & $3.7_{0^{\mathrm{a}}}$ & $3.2^{\mathrm{b}}$ & $5.5^{\mathrm{c}}$ & - & - \\
\hline
\end{tabular}

a From sedimentation equilibrium measurements.

b Taken from Part $X^{11}$ except for sample E-4.

c From osmotic pressure measurements.

Numerical values of $\bar{M}_{w}, A_{2}$, and $\left\langle S^{2}\right\rangle^{1 / 2}$ obtained in these ways from measurements in DMF at $25^{\circ} \mathrm{C}$ are collected in Table II. The last column lists the values of $h$, the pitch per monomeric residue, calculated from $\bar{M}_{w}$ and $\left\langle S^{2}\right\rangle$, with the straight rod being postulated for the molecular shape of PBLG in this solvent. They are definitely smaller than $1.5 \AA$ characteristic of the rigid intact $\alpha$-helix, and have a 


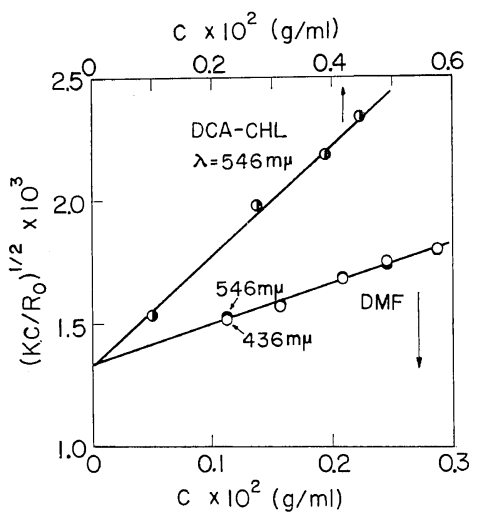

Figure 6. Plots of $\left(K c / R_{0}\right)^{1 / 2} v s . c$ for samples E-1 in DMF and in the DCA-CHL mixture at $25^{\circ} \mathrm{C}$. (C) $\mathrm{DCA}-\mathrm{CHL}$ at $546 \mathrm{~nm}$, (O) $\mathrm{DMF}$ at $436 \mathrm{~nm}$, and (O) at $546 \mathrm{~nm}$.

trend of decreasing with increasing $\bar{M}_{w}$. This result confirms those found by previous authors..$^{5-9}$ Interpretations for it will be discussed in a later section.

Figure 6 compares plots of $\left(K c / R_{0}\right)^{1 / 2} v s . c$ for sample E-1 in the DCA-CHL mixture at $25^{\circ} \mathrm{C}$ with those for the same sample in DMF at the same temperature. The data with the mixture are for light of wavelength $546 \mathrm{~nm}$, and those with DMF are for two wavelengths 436 and $546 \mathrm{~nm}$. It is observed that the plots obtained under different experimental conditions are extrapolated to an identical intercept, yielding the same value for $\bar{M}_{w}$. The same was true for sample F-2. These results confirm a similar observation with this solvent system by Strazielle, et al. ${ }^{4}$ Thus, we may expect that other molecular parameters can be obtained from the data for the DCA-CHL mixture without being disturbed by the preferential adsorption of DCA molecules onto the polypeptide chain.

Particle scattering functions $\boldsymbol{P}(\theta)$ for sample E-1 in the DCA-CHL mixture at various temperatures appear plotted in Figure 7, where the data for this sample in DMF at $25^{\circ} \mathrm{C}$ are included for comparison. The data points for the mixed solvent are fitted by a straight line, ragardless of the temperature of measurement. The slope of the line increases with rising temperature, which implies that the molecular radius of the polypeptide becomes larger as the

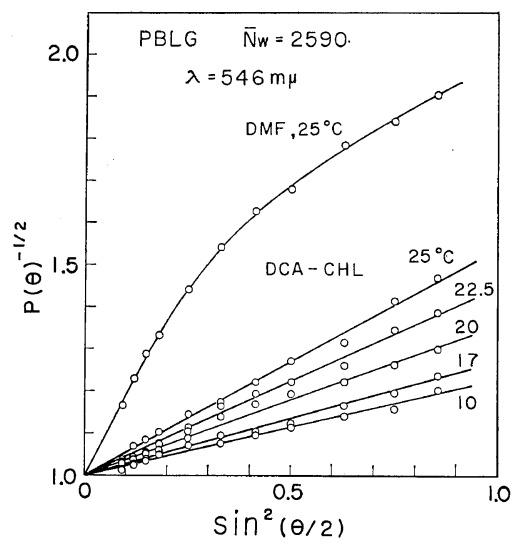

Figure 7. Representative plots of $P(\theta)^{-1 / 2} v s$. $\sin ^{2}(\theta / 2)$ for sample E-1 in the DCA-CHL mixture and in DMF at the indicated wavelength and temperatures.

temperature is raised. This behavior is a manifestation of the thermally-induced conversion of the chain from random coil to helical conformation. This fact can be seen more clearly in Figure 8 , in which the values of $\left\langle S^{2}\right\rangle^{1 / 2}$ for samples E-1 and F-2 in the DCACHL mixture are plotted against temperature, together with the values for the same samples in DMF at $25^{\circ} \mathrm{C}$; here the latter values may be regarded as the ones for essentially perfect helices. It is important to observe that, even

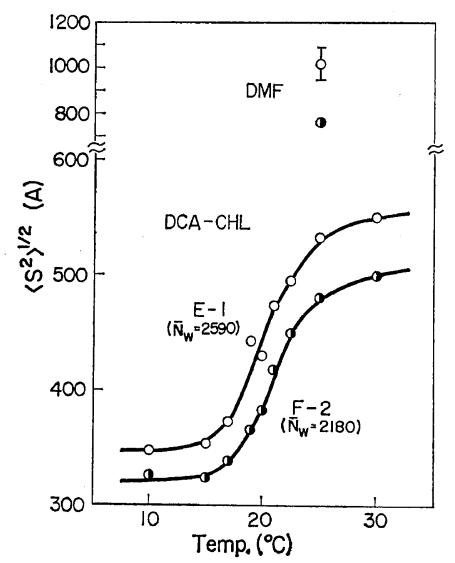

Figure 8. Temperature dependence of $\left\langle S^{2}\right\rangle^{1 / 2}$ for samples E-1 (O) and F-2 (O) in the DCA-CHL mixture and in DMF. $\bar{N}_{w}$ denotes the weightaverage degree of polymerization. 
at the highest temperature studied, the value of $\left\langle S^{2}\right\rangle^{1 / 2}$ for each sample in the mixed solvent is much smaller than that in DMF. The implication is that the transition with respect to molecular dimensions is still on its half way to helix at such a temperature. Our curve for sample E-1 bears close resemblance to that reported for a PBLG sample $\left(\bar{M}_{w}=58 \times 10^{4}\right)$ in a DCA-cyclohexane mixture by Strazielle, et al., ${ }^{4}$ with the exception that these authors observed a shallow minimum in the neighborhood of the transition temperature. Values of $\left\langle S^{2}\right\rangle^{1 / 2}$ and $A_{2}$ for samples E-1 and F-2 in the DCA-CHL mixture are collected in Table III.

Table III. Numerical results of $\langle S\rangle^{1 / 2}$ and $A_{2}$ for PBLG E-1 and F-2 in DCA containing 8.3-wt\% cyclohexanol

\begin{tabular}{|c|c|c|c|c|}
\hline \multirow{2}{*}{$\underset{{ }^{\circ} \mathrm{C}}{\mathrm{Temp}}$} & \multicolumn{2}{|c|}{ E-1 } & \multicolumn{2}{|c|}{ F-2 } \\
\hline & $\begin{array}{c}\left\langle S^{2}\right\rangle^{1 / 2} \\
\AA\end{array}$ & $\begin{array}{c}A_{2} \times 10^{4} \\
\mathrm{ml} \mathrm{mol} / \mathrm{g}^{2}\end{array}$ & $\left\langle S^{2}\right\rangle_{\AA}^{1 / 2}$, & $\begin{array}{c}A_{2} \times 10^{4} \\
\mathrm{ml} \mathrm{mol} / \mathrm{g}^{2}\end{array}$ \\
\hline 10 & $34_{7}$ & $3.3_{2}$ & $32_{6}$ & $3.3_{0}$ \\
\hline 15 & $35_{3}$ & $3.6_{2}$ & $32_{4}$ & $3.2_{2}$ \\
\hline 17 & $37_{2}$ & $3.2_{5}$ & $33_{8}$ & $3.2_{7}$ \\
\hline 19 & $44_{2}$ & $2.8_{4}$ & $36_{6}$ & $3.4_{5}$ \\
\hline 20 & $43_{0}$ & $3.1_{4}$ & $38_{2}$ & $2.7_{7}$ \\
\hline 21 & $47_{3}$ & $3.2_{5}$ & $41_{7}$ & $2.8_{8}$ \\
\hline 22.5 & $49_{5}$ & 2.89 & 449 & $2.8_{8}$ \\
\hline 25 & $53_{2}$ & $2.9_{7}$ & $48_{0}$ & $2.8_{5}$ \\
\hline 30 & $55_{0}$ & $2.7_{6}$ & $49_{9}$ & $2.5_{7}$ \\
\hline
\end{tabular}

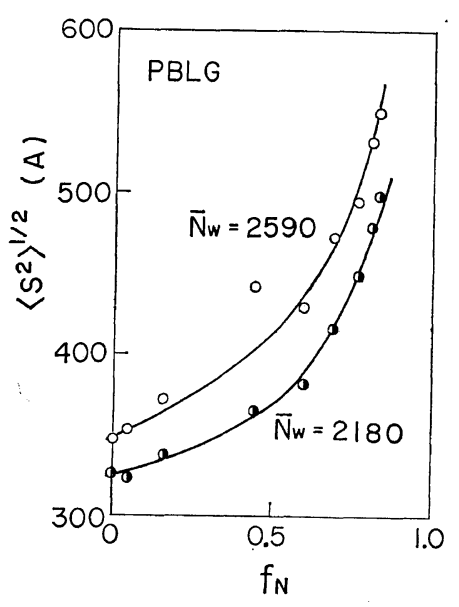

Figure 9. Variation of $\left\langle S^{2}\right\rangle^{1 / 2}$ with helical content $f_{N}$ for samples E-1 $(O)$ and F-2 (O) in the DCA-CHL mixture.
The data are combined with the corresponding data for $f_{N}$ as a function of temperature (obtained from the measurements of $[\alpha]_{546}$ ) to obtain the relation between $\left\langle S^{2}\right\rangle^{1 / 2}$ and $f_{N}$. The results are shown in Figure 9. The curves have essentially similar features to those reported for PHPG in water-methanol mixtures by Okita, et al. ${ }^{1}$

Finally, we wish to mention something about the light-scattering measurements conducted with solutions of samples E-1 and F-2 in mixtures of DCA and EDC (65 and 70 vol $\%$ of DCA) and in pure DCA. The data for these mixtures exhibited an unusually small or almost vanishing angular dependence of the intensity of scattered light, both at 436 and $546 \mathrm{~nm}$, and yielded absurdly small molecular weights and dimensions. These findings are consistent with the observations by Strazielle, et al., ${ }^{4}$ who studied a PBLG sample in a DCA-EDC mixture of similar composition with light of wavelength $546 \mathrm{~nm}$. They attributed the anomalies to the preferential adsorption of DCA molecules, but this possibility may be ruled out, since we observed similar phenomena even in pure DCA. Further investigations of this problem are highly desirable.

\section{DISCUSSION}

Analysis of the Data for $\left\langle S^{2}\right\rangle$ in the DCA-CHL Mixture

As has been shown in Part VII, ${ }^{3}$ Nagai's expression for $\left\langle S^{2}\right\rangle$ of an interrupted helix can be simplified, under appropriate conditions for $N$ (degree of polymerization of the polypeptide) and $\sigma$, to give

$$
H_{1} / H_{2}=b_{0}{ }^{2}+b_{1}{ }^{2}\left(H_{3} / H_{2}\right)
$$

where

$$
\begin{aligned}
H_{1} & =6\left\langle S^{2}\right\rangle / N(1-f) \\
H_{2} & =1+\frac{12 f^{2}}{\beta^{2}}\left[1-\frac{2 \sqrt{f(1-f)}}{\beta}\right] \\
H_{3} & =\frac{2}{\sqrt{\sigma}}\left(\frac{f}{1-f}\right)^{3 / 2} Q(\beta, f)
\end{aligned}
$$

and

$$
\beta=N \sqrt{\sigma}
$$


The expression for $Q(\beta, f)$ is lengthy, so the reader should consult Part $\mathrm{VII}^{3}$ for it. The quantities $b_{0}$ and $b_{1}$ have a physical significance such that the former is the effective bond length of randomly coiled portions in the interrupted helix and the latter is the pitch per monomer residue in its helical portions.

If both $b_{0}$ and $b_{1}$ are constant in the substantial range of the transition from random coil to helix, it follows from eq 2 that a plot of $H_{1} / H_{2}$ against $H_{3} / H_{2}$ should give a straight line, regardless of the molecular weight of the polypeptide sample, and that one can evaluate $b_{0}$ and $b_{1}$ from the intercept and slope of the line. With the numerical data for samples E-1 and F-2 in the DCA-CHL mixture (Tables I and III), we computed $H_{1} / H_{2}$ and $H_{3} H_{2}$ and obtained the plot as shown in Figure 10. As expected, the plotted points obey a linear relation, giving $1.5_{3} \pm 0.10 \AA$ for $b_{1}$. The intercept of the line indicated is too small to be determined with precision. Therefore, we estimated $b_{0}$ from the $\left\langle S^{2}\right\rangle$ values actually obtained at $10^{\circ} \mathrm{C}$, at which the helical content was essentially zero for either sample, and obtained $b_{0}=16.9 \pm 0.2 \AA$.

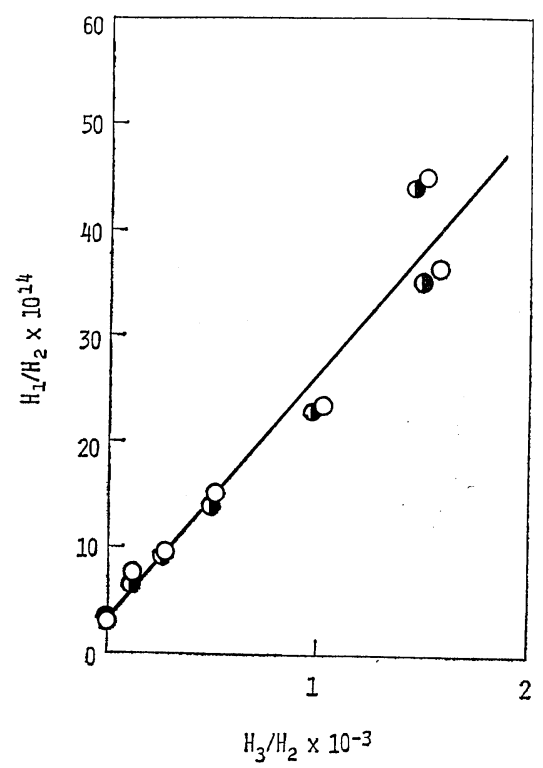

Figure 10. Analysis of $\left\langle S^{2}\right\rangle$ data for PBLG in the DCA-CHL mixture in terms of the $H_{1} / H_{2}$ vs. $H_{3} H_{2}$ plot (eq 2). The symbols are the same as those in Figure 9.
This $b_{0}$ value is in agreement with $16.7 \AA$, which can be calculated from the data of Strazielle, et al., ${ }^{4}$ for a PBLG sample $\left(\bar{M}_{w}=58 \times 10^{4}\right)$ in a DCA-cyclohexane mixture at $15^{\circ} \mathrm{C}$.

These results are similar to those derived by Okita, et al., ${ }^{1}$ for PHPG in water-methanol mixtures. Our value of $b_{1}$ again compares favorably to $1.5 \AA$ expected for the $\alpha$-helix. The disparity of our $b_{0}$ value from the BrantFlory ${ }^{16}$ value for unperturbed polypeptide chains $(11.3 \AA)$ also may be attributed to the excludedvolume effect, as has been advocated by Okita, et al., in their paper on PHPG.

The linear relation displayed in Figure 10 does not necessarily require that the parameter $b_{0}$ be strictly constant through the transition region. As can be seen, the intercept of the plot is so small that the first term on the righthand side of eq 2 is almost negligible in comparison with the second term except at very small values of $\mathrm{H}_{3} / \mathrm{H}_{2}$.

Accordingly, one would obtain an equally good straight line if $b_{0}$ varied rather appreciably by the excluded-volume effect. All that we need for a straight line to be obtained in the systems studied is that the parameter $b_{1}$ remains constant over the helix-coil transition region, or more specifically speaking, for values of $f$ larger than 0.5 , because the plot of this type is governed overwhelmingly by the data for such relatively high helical contents.

In sum, our light-scattering measurements on PBLG in the DCA-CHL mixture lend experimental support to the validity of Nagai's theory ${ }^{2}$ for the molecular dimensions of the interrupted helix.

\section{The Helix Pitch as a Function of Molecular Weight}

The values obtained for the helix pitch $h$ of PBLG in DMF at $25^{\circ} \mathrm{C}$ are plotted against $\bar{M}_{w}$ in Figure 11, wherein the data reported by previous authors ${ }^{6-9}$ for the same polypeptide in DMF and in EDC are also shown for comparison. The plotted points are in considerable scatter, reflecting the difficulty of measuring molecular dimensions of a rodlike macromolecule, on one hand, and the possible difference in polydispersity among the samples used by different authors, on the other hand. 


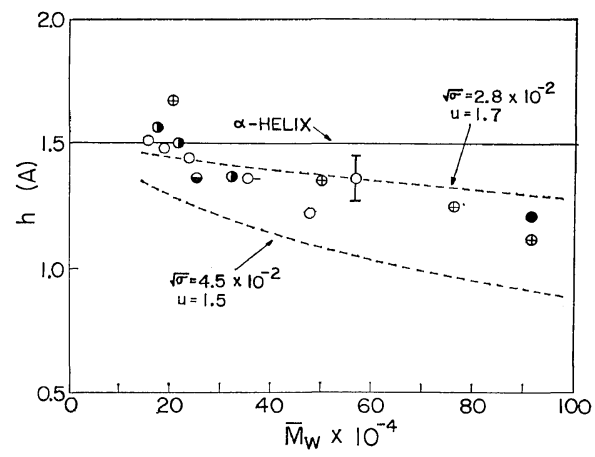

Figure 11. Molecular weight dependence of the pitch per monome.ic residue, $h$, for PBLG in DMF and EDC derived from light-scattering measurements. Data in DMF: (O) present work, (O-) Fujita, et al.,8 (reanalyzed by the procedure described in the text), and $(\oplus)$ Moha et al., ${ }^{6}$ Data in EDC: (-) Moha, et al., ${ }^{6}$ ( W) Wallach and Benoit, ${ }^{7}$ and (-) Jennings and Jerrard. ${ }^{9}$ The solid line denotes the value expected for the $\alpha$-helix, and the dashed lines represent the theoretical values which were calculated from Nagai's expression $^{2}$ for $\left\langle S^{2}\right\rangle$ by using the respective sets of the transition parameters indicated (see text).

Nevertheless, these data lead to the belief that the quantity $h$ calculated from the observed $\left\langle S^{2}\right\rangle$ and $\bar{M}_{w}$ with the assumption of a straight rod for the molecule decreases monotonically as $\bar{M}_{w}$ becomes higher. This trend, if real, implies either (1) that the $\alpha$-helix formed by PBLG in helicogenic solvents is intact but has a certain flexibility as a whole or (2) that the helix contains a small number of defects in which a few monomer units are not completely hydrogen bonded with the neighboring units and thus allow the molecular rod to bend at their position. In the case (1), the molecule is compatible with the wormlike chain, whereas in the case (2), the molecule is a limiting form of the interrepted helix in which the content of randomly coiled portions is very small.

Some groups of authors ${ }^{6,7,17-18}$ attempted to use the wormlike chain model in the analysis of light-scattering and hydrodynamic data for polypeptides in helicogenic solvents. However, they were led to a conclusion inconsistent with the $\alpha$-helix. This appears to be the most serious argument against the wormlike chain model for polypeptides, since one now has much evidence for the $\alpha$-helical conformation in solution. ${ }^{17,20,21}$

On the other hand, Miller and Flory ${ }^{10}$ have suggested the use of interrupted helices, presenting several sets of values of $\sigma$ and $u$ which accounted for experiment satisfactorily. In this case, a question arises whether the values chosen for their numerical computation correspond to the actual situation with PBLG or other polypeptides in helicogenic solvents. The direct answer can be obtained by studying the way in which the helical content of a given polypeptide varies with the molecular weight of the sample, especially in the range of low molecular weight. In fact, as will be reported elsewhere, ${ }^{22}$ one of the present authors has analyzed ORD data for a series of low molecular-weight PBLG samples in DMF of $20^{\circ} \mathrm{C}$ by Nagai's theory, and obtained $u=1.5 \pm 0.1$ and $\sqrt{\sigma}=(4.5 \pm 1.0)$. $\times 10^{-2}$ as rough estimates.

In Part XI, Sayama, et al., ${ }^{15}$ proposed an indirect means whereby the values of $u$ and $\sigma$ are estimated from polarimetric data for mixed solvents at various compositions, although their theory was formulated on a certain specific mechanism for the binding of the helix-breaking solvent on the polypeptide chain. From the analysis of the data for DCA-EDC mixtures, they obtained $\sqrt{\sigma}=2.8 \times 10^{-2}$ and $u=1.7$ for PBLG in pure EDC at $25^{\circ} \mathrm{C}$. This set of values compares favorably to that obtained directly for DMF, and is consistent with those which were assigned by Miller and Flory ${ }^{10}$ in their comparison of theory and experiment.

The dashed lines in Figure 11 represent the theoretical values corresponding to these two sets of values. The data points fall in the region between the two lines, and agreement of theory and experiment may be regarded as satisfactory. Calculations showed that, over the range of $\bar{M}_{w}$ above $10^{5}$, the helical contents were not different from unity more than $0.6 \%$ with the first set of the transition parameters and $0.1 \%$ with the second set. This implies that under the experimental conditions in which the data points shown in Figure 11 were obtained, the polypeptide samples used were in the state which was polarimetrically indistinguishable from the perfect helix.

Finally, we calculate, by using Nagai's expression, the average number of helical sections. 
in the chain for the case $u=1.7$ and $\sqrt{\sigma}=2.8$ $\times 10^{-2}$. Then we obtain 1.2 at $N=500,1.4$ at $N=1000,2.2$ at $N=3000$. Thus, on the average, the PBLG chain having an $N$ of $2560\left(56 \times 10^{4}\right.$ in terms of molecular weight) assumes the form of a once-broken rod when dissolved in EDC. It can be shown that if the break in such a rod is completely flexible and occurs at any position with equal probability, the chain is characterized by an $h$ of $1.34 \AA$. Interestingly, this value is in close agreement with the observed $h$ for sample E-1 and also with the caluclated one which is read off the dashed line (Figure 11) at $\bar{M}_{w}=56 \times 10^{4}$.

\section{REFERENCES}

1. K. Okita, A. Teramoto, and H. Fujita, Polymer J., 1, 582 (1970).

2. K. Nagai, J. Chem. Phys., 34, 887 (1961).

3. A. Teramoto, T. Norisuye, and H. Fujita, Polymer J., 1, 55 (1970).

4. C. Strazielle, C. Dufour, and E. Marchal, Eur. Polym. J., 6, 1133 (1970).

5. P. Doty, J. H. Bradbury, and A. M. Holtzer, J. Amer. Chem. Soc., 78, 947 (1956).

6. P. Moha, G. Weill, and H. Benoit, J. Chim. Phys., 61, 1240 (1964).

7. M. Wallach and H. Benoit, J. Polym. Sci., 57, 41 (1962).

8. H. Fujita, A. Teramoto, K. Okita, T. Yamashita, and S. Ikeda, Biopolymers, 4, 769 (1966).
9. B. R. Jennings and H. G. Jerrard, J. Phys. Chem., 69, 2817 (1965).

10. W. G. Miller and P. J. Flory, J. Mol. Biol., 15, 298 (1966).

11. T. Norisuye, M. Matsuoka, A. Teramoto, and H. Fujita, Polymer J., 1, 691 (1970).

12. T. Norisuye, Ph. D. Thesis, Department of Polymer Science, Osaka University, 1973.

13. Gj. Dezělić and J. Vavra, Croat. Chim. Acta, 38, 35 (1966).

14. K. Okita, A. Teramoto, and H. Fujita, Biopolymers, 9, 717 (1970).

15. N. Sayama, K. Kida, T. Norisuye, A. Teramoto, and H. Fujita, Polymer J., 3, 538 (1972).

16. D. A. Brant and P. J. Flory, J. Amer. Chem. Soc., 87, 2788 (1965).

17. J. Applequist and P. Doty, in "Polyamino Acids, Polypeptides, and Proteins," M. A. Stahmann Ed., Univ. Wisconsin Press, Madison, 1962, p 161.

18. H. Benoit, L. Freund, and G. Spach, in "Poly$\alpha$-Amino Acids," G. D. Fasman Ed., Marcel Dekker, Inc., New York, 1967, p 105.

19. V. N. Tsvetkov, Yu. V. Mitin, I. N. Shtennikova, V. R. Glushenkova, G. V. Tarasova, V. S. Skazka, and N. A. Nikitin, Vysokomol. Soedin., 7, 1098 (1965).

20. D. A. D. Parry and A. Elliott, Nature, 206, 616 (1965).

21. P. Saludjian and V. Luzzati, J. Mol. Biol., 15, 681 (1966).

22. A. Teramoto, to be published. 